\title{
Fatores determinantes do envelhecimento saudável em idosos residentes em centro urbano: Projeto Epidoso, São Paulo
}

\author{
Determinant factors for healthy aging among \\ senior citizens in a large city: the Epidoso \\ Project in São Paulo
}

Luiz Roberto Ramos 1

\footnotetext{
1 Departamento de Medicina,

Centro de Estudos do Envelhecimento, Universidade Federal de São Paulo. Rua dos Ottonis 731, São Paulo, SP 04025-002, Brasil. luizramos.dmed@epm.br
}

\begin{abstract}
Population aging leads to an increase in the prevalence of chronic and disabling diseases, as well as a change in the public health paradigm. Diseases diagnosed in the elderly are generally not curable; if not properly treated and monitored over time, they tend to generate complications and sequelae that impair patients' independence and autonomy. Health is no longer measured by the presence or absence of disease, but by the degree of preservation of functional capacity. Factors for healthy aging with good functional capacity and those which increase the risk of death and disability need to be identified by longitudinal surveys that include the elderly population living in the community. This article presents data from the first followup survey of senior citizens in Brasil, called the Epidoso Project (from "epi" as in "epidemiologic" and "idoso" or "elderly" in Portuguese) implemented since 1991 in the city of São Paulo. The socio-demographic, clinical, and functional characteristics of a cohort of elderly are discussed, with a risk analysis for death and disability, and the implications for health planning are considered.
\end{abstract}

Key words Aging Health; Demographic Aging; Chronic Disease; Longitudinal Studies

Resumo Com o envelhecimento populacional, temos um aumento da prevalência de doenças crônicas e incapacitantes e uma mudança de paradigma na saúde pública. As doenças diagnosticadas num indivíduo idoso geralmente não admitem cura e, se não forem devidamente tratadas e acompanhadas ao longo dos anos, tendem a apresentar complicações e seqüelas que comprometem a independência e a autonomia do paciente. A saúde não é mais medida pela presença ou não de doenças, e sim pelo grau de preservação da capacidade funcional. Quais os fatores que determinam um envelhecimento saudável, com boa capacidade funcional, e quais os fatores que aumentam o risco de morte e incapacidade são questões que terão que ser respondidas por inquéritos longitudinais que incluam a população idosa residente na comunidade. Este artigo apresenta dados do primeiro inquérito populacional de seguimento com idosos na comunidade no Brasil - Projeto Epidoso, em curso desde 1991 na cidade de São Paulo. São discutidas as características sócio-demográficas, clínicas e funcionais de uma coorte de idosos, com análise de risco para morte e incapacidade e considerações sobre as implicações para o planejamento em saúde.

Palavras-chave Saúde do Idoso; Envelhecimento da População; Doença Crônica; Estudo Longitudinal 


\section{Capacidade funcional: um novo paradigma em saúde}

Embora a grande maioria dos idosos seja portadora de, pelo menos, uma doença crônica (Ramos et al., 1993), nem todos ficam limitados por essas doenças, e muitos levam vida perfeitamente normal, com as suas enfermidades controladas e expressa satisfação na vida. Um idoso com uma ou mais doenças crônicas pode ser considerado um idoso saudável, se comparado com um idoso com as mesmas doenças, porém sem controle destas, com seqüelas decorrentes e incapacidades associadas. Assim, o conceito clássico de saúde da Organização Mundial da Saúde (OMS) mostra-se inadequado para descrever o universo de saúde dos idosos, já que a ausência de doenças é privilégio de poucos, e o completo bem-estar pode ser atingido por muitos, independentemente da presença ou não de doenças.

Na verdade, o que está em jogo na velhice é a autonomia, ou seja, a capacidade de determinar e executar seus próprios desígnios. Qualquer pessoa que chegue aos oitenta anos capaz de gerir sua própria vida e determinar quando, onde e como se darão suas as atividades de lazer, convívio social e trabalho (produção em algum nível) certamente será considerada uma pessoa saudável. Pouco importa saber que essa mesma pessoa é hipertensa, diabética, cardíaca e que toma remédio para depressão - infelizmente uma combinação bastante freqüente nessa idade. O importante é que, como resultante de um tratamento bem-sucedido, ela mantém sua autonomia, é feliz, integrada socialmente e, para todos os efeitos, uma pessoa idosa saudável.

Uma outra pessoa com a mesma idade e as mesmas doenças, porém sem controle destas, poderá apresentar um quadro completamente diferente. Inicialmente sob a influência da depressão, essa pessoa poderá apresentar uma progressiva reclusão social, com tendência ao sedentarismo, déficit cognitivo, perda de autoestima e abandono de autocuidados. Paralelamente, o diabetes e o problema cardíaco, que de início não limitavam, passam a limitar fisicamente, agravando o problema mental e aumentando o risco para complicações cardiovasculares. Nesse momento a capacidade funcional encontra-se já bastante comprometida, com dependência física e mental para a realização de atividades da vida diária mais complexas, como, por exemplo, limpar a casa, fazer compras, cuidar das finanças. No momento seguinte, o advento de um acidente vascular cerebral ou infarto do miocárdio não fatais pode reme- ter essa pessoa para um novo patamar de dependência, no qual será necessário assistência continuada para a realização das atividades mais básicas da vida cotidiana, como comer, vestir, ou tomar banho. Eventualmente, o adequado tratamento dessas doenças pode reverter o quadro, mas não a ponto de retornar ao patamar inicial. Nesse caso, ninguém hesitaria em caracterizar essa pessoa como doente.

Capacidade funcional surge, portanto, como um novo paradigma de saúde, particularmente relevante para o idoso (Fillenbaum, 1984; Kane \& Kane, 1981). Envelhecimento saudável, dentro dessa nova ótica, passa a ser a resultante da interação multidimensional entre saúde física, saúde mental, independência na vida diária, integração social, suporte familiar e independência econômica. A perda de um ente querido, a falência econômica, uma doença incapacitante, um distúrbio mental, um acidente, são eventos cotidianos que podem, juntos ou isoladamente, comprometer a capacidade funcional de um indivíduo. O bem-estar na velhice, ou saúde num sentido amplo, seria o resultado do equilíbrio entre as várias dimensões da capacidade funcional do idoso, sem necessariamente significar ausência de problemas em todas as dimensões.

\section{Avaliação multidimensional do idoso}

Com base no conceito de saúde do idoso como capacidade funcional, foram desenvolvidos inúmeros instrumentos abrangendo as várias dimensões pertinentes à avaliação global da capacidade funcional de um idoso. Um dos primeiros instrumentos deste tipo foi o Olders Americans Research and Services (OARS), Multidimensional Functional Assessment Questionnaire (OMFAQ), concebido nos Estados Unidos (Duke University Center for the Study of Aging and Human Development, 1978) e traduzido e adaptado para o português - BOMFAQ (Brazilian version of OMFAQ) (Ramos \& Goihman, 1989). Trata-se de um questionário fechado que fornece dados sócio-demográficos, avalia a percepção subjetiva do idoso, a saúde física e mental (aspectos cognitivos e emocionais), independência no dia-a-dia, suporte social e familiar e utilização de serviços. O objetivo desse instrumento é proporcionar um perfil de saúde multidimensional, identificando quais as dimensões que mais diretamente comprometem a capacidade funcional da população e, com isso, indicando soluções que transcendem uma linha programática baseada no aumento da cobertura diagnóstica e terapêutica das doenças 
crônicas não transmissíveis (DCNT) que acometem o idoso.

Um dos primeiros estudos populacionais, com avaliação multidimensional de idosos residentes na comunidade, no Brasil, foi realizado na cidade de São Paulo em 1984 (Ramos \& Goihman, 1989). Os dados desse estudo mostraram que o idoso residente num grande centro urbano latino-americano apresentava um perfil muito semelhante ao que se esperaria de uma população de idosos residente em um país desenvolvido, porém com algumas idiossincrasias marcantes.

Em geral, a população de idosos apresentou uma alta prevalência de doenças crônicas quase $90 \%$ referiram pelo menos uma DCNT -, principalmente hipertensão arterial, dores articulares e varizes. Quase a metade referiu precisar de ajuda para realizar pelo menos uma das atividades da vida diária, instrumentais ou pessoais (limpar a casa, ir ao banheiro, comer, trocar de roupa, etc.), e cerca de um quarto teve um screening positivo para distúrbio emocional tipo distimia. Esses dados poderiam perfeitamente dizer respeito a uma população urbana européia, por exemplo. No entanto, alguns dados da caracterização sócio-demográfica, em particular, eram bastante distintos.

A começar pela idade média (72 anos), que era baixa quando comparada à de países desenvolvidos, e pela razão de homens/mulheres, que foi mais elevada do que em países desenvolvidos, onde a proporção de mulheres é bastante superior à de homens. Todavia, as duas variáveis que se mostraram mais diferenciadas de um padrão europeu ou norte-americano foram o nível sócio-econômico e o arranjo domiciliar do idoso.

A renda média per capita no domicílio do idoso da amostra foi de US\$100/mês, variando de US\$32, no subdistrito periférico de baixa renda, a US\$233. no subdistrito central de alta renda. O nível de renda mostrou-se fortemente associado com a saúde física e mental. A proporção de idosos com DCNT, ou depressão, ou dependência no dia-a-dia foi significativamente mais alta entre idosos de baixa renda.

O arranjo domiciliar, por sua vez, mostrou que o idoso nessa região do Brasil ainda vive majoritariamente dividindo o domicílio com seus filhos e muitas vezes com filhos e netos. Esse tipo de domicílio, chamado multigeracional, acomodava mais de $50 \%$ dos idosos, e em metade das residências o idoso vivia com filhos casados e com netos. Esse achado contrastava com o que se verifica em países desenvolvidos, onde menos de $5 \%$ dos idosos vivem em domicílios com os filhos e muito raramente com os netos. A maioria vive com o cônjuge apenas, ou só. Em São Paulo, apenas $10 \%$ dos idosos viviam sós, menos de um terço do que se espera em qualquer população européia, por exemplo. Os dados aparentemente confirmaram a concepção de que os idosos no Brasil, assim como nos países latino-americanos, em geral, teriam um suporte familiar mais intenso do que os idosos na Europa, evidenciando um traço cultural, que de certa forma compensaria o desnível sócio-econômico.

Por outro lado, os diferentes tipos de arranjos domiciliares abrigavam idosos com características bastante distintas. Os idosos vivendo em domicílios com filhos e netos eram, geralmente, viúvas, bem idosas, de origem rural, com uma renda muito baixa ou inexistente. Apresentavam uma prevalência acima da média de DCNT, de dependência severa no dia-a-dia, e de distúrbio afetivo, como depressão. Apesar da presença física de vários familiares, eram idosos que, com uma freqüência acima da média, referiam sensação de solidão.

O idoso morando apenas com filhos era, em geral, do sexo masculino, de origem rural, casado, mais jovem, com melhor condição sócioeconômica e mais independência no dia-a-dia. No caso dos domicílios em que vivia apenas um casal, o idoso era geralmente homem, de origem urbana, também no grupo etário mais jovem, com poucas doenças crônicas e total independência no dia-a-dia.

Os idosos vivendo sós eram, em geral, mulheres com uma origem urbana, viúvas, de nível sócio-econômico baixo, com várias doenças e um nível intermediário de dependência no dia-a-dia, já que os estados de dependência grave mostraram-se incompatíveis com a vida só. Alternativamente, essa idosa morando só poderia ser uma pessoa solteira, com renda pessoal mais alta e condição de saúde e independência acima da média.

A conclusão a que se chegou foi que os arranjos domiciliares multigeracionais, além de serem extremamente prevalentes, associavamse significativamente com um nível sócio-econômico baixo, geralmente afetando mulheres viúvas com várias doenças e um grau de moderado a severo de dependência no dia-a-dia. Mais do que uma opção sócio-cultural, tais arranjos mostraram-se uma forma de sobrevivência. Na verdade, os idosos com nível sócioeconômico mais alto viviam majoritariamente apenas com o cônjuge ou sós, reproduzindo o modelo verificado nos países mais desenvolvidos. Vale referir que nesses casos não havia, necessariamente, uma falta de suporte familiar; havia, sim, um esquema de intimidade a dis- 
tância entre os membros da família, nos moldes que se verificam nos países mais desenvolvidos (Ramos, 1992; Ramos et al., 1992).

\section{Fatores de risco para mortalidade}

A velhice é um período da vida com uma alta prevalência de DCNT, limitações físicas, perdas cognitivas, sintomas depressivos, declínio sensorial, acidentes e isolamento social. Entretanto, tem crescido o interesse em estabelecer quais os fatores que, isolada ou conjuntamente, melhor explicam o risco que um idoso tem de morrer em curto prazo, uma noção útil do ponto de vista epidemiológico e clínico. Para responder a essa questão, no entanto, faz-se necessário estudos longitudinais, que acompanhem coortes de idosos, controlando os possíveis fatores de risco.

Estudos longitudinais com amostras populacionais, especialmente desenhados para avaliar fatores de risco para mortalidade em idosos, ainda são relativamente raros na literatura. Alguns estudos discutem fatores de risco para mortalidade em idosos, mas baseiam-se em amostras não populacionais, ou analisam amostras populacionais que não foram originalmente selecionadas para estudar idosos.

O principal fator de risco para mortalidade continua sendo a própria idade. Quanto mais se vive maior é a chance de morrer. A maioria dos estudos longitudinais com idosos residentes na comunidade parece concordar que, além da idade, o sexo do indivíduo pode ser determinante do risco morte, com os homens apresentando um risco maior do que as mulheres (Jagger et al., 1993; NCHS, 1985). Todas as demais variáveis são dependentes de uma complexa interação entre o indivíduo e o meio ambiente, que, por sua vez, varia de cultura para cultura e de tempos em tempos. Mesmo o fator sexo pode vir a ter sua relação de risco alterada no futuro, com a evolução social promovendo um aumento significativo de mortes por DCV entre as mulheres, agora mais expostas do que antes aos fatores de risco ocupacionais e ambientais, devido a sua progressiva incorporação à população economicamente ativa.

Outros fatores objetivos da avaliação multidimensional de pessoas idosas os quais gozam de uma concordância entre os vários estudos são o grau de incapacidade, avaliado pelo desempenho nas atividades da vida diária (AVDs), e história de hospitalização prévia no último ano.

Uma variável que tem merecido considerável atenção nessa área de determinantes de mortalidade entre idosos é a auto-avaliação subjetiva de saúde do idoso. Por ser uma variável simples de ser obtida, com potencial de sintetizar uma complexa interação de fatores envolvidos na saúde de um idoso, e com alto valor preditivo de mortalidade, a maioria dos estudos dedica atenção especial à discussão das implicações práticas dessa variável como um indicador de saúde (Mossey \& Shapiro, 1982). Entretanto, poucos estudos incluíram a medida do estado cognitivo do idoso entre as variáveis independentes do modelo multivariado de determinação de risco de morte, e praticamente nenhum estudo avaliou o grau de depressão do entrevistado. Ambas as variáveis podem, em teoria, confundir a associação entre auto-avaliação de saúde e o risco de morte entre idosos.

Epistemologicamente a morte vem associada com a doença física, numa concepção, em geral válida, de que as pessoas doentes morrem mais. Contudo, os estudos sobre determinantes de mortalidade em idosos têm mostrado que as doenças crônicas referidas ou diagnosticadas não afetam significativamente o risco de morte (Wolinsky et al., 1995). Provavelmente devido à variabilidade com que a mesma doença afeta a capacidade funcional.

Vale lembrar, no entanto, que nenhum dos estudos longitudinais, acima referidos, sobre risco de morte em idosos foi conduzido em país em desenvolvimento, onde a pobreza, o nível educacional baixo e uma estrutura familiar diferenciada podem introduzir variações idiossincráticas no modelo preditivo de morte.

O Projeto Epidoso é o primeiro estudo longitudinal com idosos, na América Latina, que avaliou pessoas residentes na comunidade, com um instrumento multidimensional e seguiu a coorte em busca de fatores associados ao envelhecimento saudável e fatores de risco para mortalidade. Há mais de dez anos, pessoas que tinham 65 anos ou mais em 1991 são seguidas no domicílio e acompanhadas em ambulatório, na área de captação do Centro de Estudos do Envelhecimento (CEE) da Escola Paulista de Medicina, Universidade Federal de São Paulo (EPM/UNIFESP), no Município de São Paulo (Ramos et al., 1998).

A mortalidade por todas as causas nessa coorte de idosos residentes em zona urbana foi de quase $10 \%$ em dois anos. Os fatores que aparentemente influenciaram o risco de morte, resultado de uma análise bivariada, foram: sexo (masculino), idade (avançada), auto-avaliação subjetiva de saúde (negativa), história pregressa de sedentarismo, edentulismo, quedas, acidente vascular cerebral e incontinência urinária, hospitalização e visita ao médico nos últi- 
mos seis meses, positividade para depressão, déficit cognitivo e dependência no dia-a-dia.

Utilizando-se um modelo multivariado de análise, com regressão logística, poucas variáveis mantiveram um efeito independente e significante no risco de morte, a saber: sexo, idade, hospitalização prévia e positividade nos rastreamentos para déficit cognitivo e dependência no dia-a-dia (Ramos et al., 2001).

Na prática, os únicos fatores de risco mutáveis que poderiam diminuir o risco de morte foram o estado cognitivo e o grau de dependência no dia-a-dia, avaliados, respectivamente, através de do mini-exame do estado mental (Folstein et al., 1975) e da escala de atividades da vida diária (Ramos et al., 1993), ambas escalas simples, confiáveis e validadas, que poderiam ser incorporados ao protocolo clínico de atenção à saúde do idoso em nível primário.

\section{A saúde pública e o novo paradigma}

O desafio maior no século XXI será cuidar de uma população de mais de 32 milhões de idosos, a maioria com nível sócio-econômico e educacional baixos e uma alta prevalência de doenças crônicas e incapacitantes.

A principal fonte de suporte para essa população de idosos ainda é a família, principalmente aquela que, em domicílios multigeracionais, coabita com o idoso, o qual representa uma parcela da população de idosos que tende a ser mais pobre, com mais problemas de saúde e mais dependente no dia-a-dia do que a média dos idosos. Afora as limitações financeiras para aderir aos múltiplos tratamentos necessários, geralmente em bases crônicas, a disponibilidade de suporte familiar para o idoso dependente deverá decair marcadamente em face da diminuição do tamanho da família, o aumento do número de pessoas atingindo idades avançadas e a crescente incorporação da mulher - principal cuidadora - à força de trabalho fora do domicílio.

O sistema de saúde terá que fazer frente a uma crescente demanda por procedimentos diagnósticos e terapêuticos das doenças crônicas não transmissíveis, principalmente as cardiovasculares e as neurodegenerativas, e a uma demanda ainda maior por serviços de reabilitação física e mental. Será preciso estabelecer indicadores de saúde capazes de identificar idosos de alto risco de perda funcional e orientar ações concentradas de promoção de saúde e manutenção da capacidade funcional. Ações que tenham um significado prático para os profissionais atuando no nível primário de atenção à saúde e que tenham uma relação de custo-benefício aceitável para os administradores dos parcos recursos destinados à área da saúde.

Estudos transversais já haviam demonstrado que os idosos em um centro urbano apresentam uma alta prevalência de incapacidades físicas e mentais geradoras de dependência no dia-a-dia. O seguimento longitudinal mostrou que essas limitações aumentavam significativamente o risco de morte nessa população.

Medidas de intervenção visando identificar causas tratáveis de déficit cognitivo e de perda de independência no dia-a-dia deveriam tornar-se prioridade do sistema de saúde, dentro de uma perspectiva de reestruturação programática realmente sintonizada com a saúde e o bem-estar da crescente população de idosos. $\mathrm{O}$ objetivo principal do sistema deve ser a manutenção da capacidade funcional do idoso, mantendo-o na comunidade, pelo maior tempo possível, gozando ao máximo sua independência.

A manutenção da capacidade funcional é, em essência, uma atividade multiprofissional para a qual concorrem médicos, enfermeiras, fisioterapeutas, terapeutas ocupacionais, psicólogos e assistentes sociais. A presença desses profissionais na rede de saúde deve ser vista como uma prioridade. Contudo, para que a atenção ao idoso possa se realizar em bases interprofissionais é fundamental que se estimule a formação de profissionais treinados, mediante a abertura de disciplinas nas universidades, de residências médicas e de linhas de financiamento a pesquisas que identifiquem a área da geriatria e gerontologia. 


\section{Referências}

DUKE UNIVERSITY CENTER FOR THE STUDY OF AGING AND HUMAN DEVELOPMENT, 1978. Multidimensional Functional Assessment: The Oars Methodology. Durhan: Duke University Center for the Study of Aging and Human Development.

FILLENBAUM, G. G., 1984. The Well-Being Of The Elderly: Approaches To Multidimensional Assessment. Geneva: World Health Organization.

FOLSTEIN, M. F; FOLSTEIN, S. E. \& McHUGH, P. R., 1975. 'Mini-mental state': A practical method for grading the cognitive state of patients for the clinician. Journal of Psychiatric Research, 12:189-198.

JAGGER, C.; SPIERS, N. A. \& CLARKE, M., 1993. Factors associated with decline in function, institutionalization and mortality of elderly people. Age and Ageing, 22:190-197.

KANE, R. A. \& KANE, R. L., 1981. Assessing The Elderly: A Practical Guide To Measurement. Lexington: Lexington Books.

MOSSEY, J. M. \& SHAPIRO, E., 1982. Self-rated health: A predictor of mortality among the elderly. American Journal of Public Health, 72:800-808.

NCHS (National Center For Health Statistics), 1985. The National Health Survey Design, 1973-1984. Washington, DC: Department of Health and Social Security.

RAMOS, L. R., 1992. Family support for the elderly in São Paulo, Brazil. In: Family Support For The Elderly: The International Experience $(\mathrm{H}$. Kendig, A. Hashimoto \& L. Coppard, ed.), pp. 224-234, Oxford: Oxford University Press.

RAMOS, L. R. \& GOIHMAN, S., 1989. Geographic stratification by socio-economic status: Methodology from a household survey with elderly people in Sao Paulo, Brazil. Revista de Saúde Pública, 23: 478-492.
RAMOS, L. R.; PERRACINI, M.; ROSA, T. E. C. \& KALACHE, A., 1993. Significance and management of disability among urban elderly residents in Brazil. Journal of Cross-Cultural Gerontology, 8:313-323.

RAMOS, L. R.; ROSA, T. E. C.; OLIVEIRA, Z.; MEDINA, M. C.\& SANTOS, F. R. G., 1993. Profile of the elderly residents in Sao Paulo, Brazil: Results from a household survey. Revista de Saúde Pública, 27: 87-94.

RAMOS, L. R.; SANTOS, C. A.; ROSA, E. C. \& MANZOCHI, L. H., 1991. Perfil dos idosos residentes na comunidade no Município de São Paulo, segundo o tipo de domicílio: O papel dos domicílios multigeracionais. In: A População Idosa e o Apoio Familiar (Fundação SEADE, org.), pp. 109-129, São Paulo: Fundação SEADE.

RAMOS, L. R.; SIMÕES, E. \& ALBERT, M. S., 2001. Dependency on daily living and cognitive impairment strongly predicted mortality among urban elderly residents in Brazil: A two-year follow-up. Journal of the American Geriatric Society, 49:11681175.

RAMOS, L. R.; TONIOLO, J.; CENDOROGLO, M. S.; GARCIA, J. T.; NAJAS, M. S.; PERRACINI, M.; PAOLA, C. R.; SANTOS, F. C.; BILTON, T.; EBEL, S. J.; MACEDO, M. B.; ALMADA, C. M.; NASRI, F.; MIRANDA, R. D.; GONÇALVES, M.; SANTOS, A. L.; FARIETTA, R.; VIVACQUA, I.; ALVES, M. L. \& TUDISCO, E. S., 1998. Two-year follow-up study of elderly residents in Sao Paulo, Brazil (Epidoso Project): Metodology and preliminary results. Revista de Saúde Pública, 33:397-407.

WOLINSKY, F. D.; JOHNSON, R. L. \& STUMP, T. E., 1995. The risk of mortality among older adults over an eight-year period. Gerontologist, 35:150161.

Recebido em 10 de abril de 2002

Aprovado em 25 de junho de 2002 